\title{
PIECEWISE MONOTONE POLYNOMIAL INTERPOLATION
}

\author{
BY SAM W. YOUNG
}

Communicated by Alberto P. Calderon, April 19, 1967

The purpose of this paper is to prove the following:

THEOREM. Suppose that $n$ is a positive integer, $x_{i-1}<x_{i}$ and $y_{i-1} \neq y_{i}$, $i=1,2, \cdots, n$. Then there exists a polynomial $P$ such that $P\left(x_{i}\right)=y_{i}$, $i=0,1, \cdots, n$ and $P$ is monotone in each of the intervals $\left[x_{i-1}, x_{i}\right]$, $i=1,2, \cdots, n$.

This theorem differs from the usual polynomial interpolation theorem in that there is no mention of the degree of the polynomial. The proof presented here leaves little room for generalization from ordinary polynomials to more general systems of functions since it is essential that zeros can be prescribed.

Proof. Let $y_{0}=0$ and generality is not lost. $D=\{Q: Q$ is a polynomial and $Q(x)\left(y_{i}-y_{i-1}\right) \geqq 0$ for $\left.x_{i-1} \leqq x \leqq x_{i}, i=1,2, \cdots, n\right\}$. If $Q_{1}, Q_{2} \in D$ and $0 \leqq t \leqq 1$, then $\left[t Q_{1}(x)+(1-t) Q_{2}(x)\right]\left(y_{i}-y_{i-1}\right)$ $=t Q_{1}(x)\left(y_{i}-y_{i-1}\right)+(1-t) Q_{2}(x)\left(y_{i}-y_{i-1}\right) \geqq 0$ for $x_{i-1} \leqq x \leqq x_{i}$ and $i$ $=1,2, \cdots, n$. Therefore $D$ is a convex subset of the space $C\left[x_{0}, x_{n}\right]$. Furthermore, if $Q \in D$ and $a \geqq 0$, then $a Q \in D$.

Let $F$ be the function from $D$ into $E_{n}$ defined by

$$
F(Q)=\left\{\int_{x_{0}}^{x_{i}} Q(x) d x\right\}_{i=1}^{n} .
$$

$F$ is linear and so $F(D)$ is a convex subset of $E_{n}$. Furthermore if $z \in F(D)$ and $a \geqq 0$, then $a z \in F(D)$.

For each $i, 1 \leqq i \leqq n$, let $\phi_{i}$ denote the point of $E_{n}$ such that if $1 \leqq j<i$, then the $j$ th coordinate is zero and if $i \leqq j \leqq n$, then the $j$ th coordinate is one. Let $\lambda_{i}=\operatorname{sign}\left(y_{i}-y_{i-1}\right) \cdot \phi_{i}$ and note that $\phi_{i}=\operatorname{sign}\left(y_{i}-y_{i-1}\right) \cdot \lambda_{i}$. Any point $Z=\left(z_{1}, z_{2}, \cdots, z_{n}\right)$ has the representation $z=\sum\left(z_{i}-z_{i-1}\right) \phi_{i}$ where $z_{0}=0$. In particular, if $Q \in D$,

$$
F(Q)=\sum\left(\int_{x_{i-1}}^{x_{i}} Q(x) d x\right) \cdot \phi_{i}=\sum\left(\int_{x_{i-1}}^{x_{i}} Q(x) d x\right) \operatorname{sign}\left(y_{i}-y_{i-1}\right) \cdot \lambda_{i} .
$$

But

$$
\operatorname{sign}\left(\int_{x_{i-1}}^{x_{i}} Q(x) d x\right)=\operatorname{sign}\left(y_{i}-y_{i-1}\right)
$$


since $Q(x)\left[y_{i}-y_{i-1}\right] \geqq 0$ for all $x_{i-1} \leqq x \leqq x_{i}$. Therefore we have the equation

$$
F(Q)=\sum\left|\int_{x_{i-1}}^{x_{i}} Q(x) d x\right| \cdot \lambda_{i}
$$

If $Q \in D$ and $Q \not \equiv 0$ and we multiply $Q$ by a nonnegative polynomial $P$ which is near $\left|\int_{x_{i-1}}^{x_{i}} Q(x) d x\right|^{-1}$ between $x_{i-1}$ and $x_{i}$ and which is small elsewhere, then the product $Q P \in D$ and $F(Q P)$ is near $\lambda_{i}$. Thus each of the points $\lambda_{i}$ is a limit point of $F(D)$. An exact verification of this assertion requires a lengthy but unsophisticated calculation.

Let $y=\left(y_{1}, y_{2}, \cdots, y_{n}\right)$. We must now show that $y \in F(D)$. The points $\left\{\lambda_{i}\right\}$ form a basis for $E_{n}$ and $y=\sum\left(y_{i}-y_{i-1}\right) \cdot \phi_{i}=\sum\left(y_{i}-y_{i-1}\right)$ $\cdot \operatorname{sign}\left(y_{i}-y_{i-1}\right) \cdot \lambda_{i}=\sum\left|y_{i}-y_{i-1}\right| \cdot \lambda_{i}$ where by hypothesis, $\left|y_{i}-y_{i-1}\right|$ $>0, i=1,2, \cdots, n$. Since $\left\{\lambda_{i}\right\}$ is a basis each member of which is a limit point of $F(D)$, we can choose a basis $\left\{\theta_{i}\right\}$ from the set $F(D)$ close enough to $\left\{\lambda_{i}\right\}$ that if $y=\sum t_{i} \theta_{i}$, then $t_{i}>0, i=1,2, \cdots, n$. Let $\sigma$ $=\sum t_{i}$. By convexity of $F(D), \sigma^{-1} y=\sigma^{-1} \sum t_{i} \theta_{i}$ belongs to $F(D)$ and therefore $\sigma \sigma^{-1} y=y \in F(D)$.

Since $y \in F(D)$, there exists a $Q \in D$ such that $F(Q)=y$. Thus if $P(t)=\int_{x_{0}}^{t} Q(x) d x$, then $P(t)$ is a polynomial which satisfies the conclusion of the theorem.

We have used the Weierstrass theorem in the proof of this theorem. The Weierstrass theorem is also an immediate consequence of this interpolation theorem and so the two are equivalent.

UNIVERSITY OF UTAH 